Internist 2022 -63:658-661

https://doi.org/10.1007/s00108-022-01279-y

Angenommen: 24. Januar 2022

Online publiziert: 17. Februar 2022

(c) Der/die Autor(en) 2022

Redaktion

H. Haller, Hannover (Schriftleitung)

B. Salzberger, Regensburg

C.C. Sieber, Nürnberg

\section{Rohes Fleisch, viele Probleme: seltene Infektion bei einem Patienten nach mechanischem Klappenersatz und Lebertransplantation}

\author{
Michael Dölle ${ }^{1}$ Samira Soltani ${ }^{2} \cdot$ Heiner Wedemeyer ${ }^{1} \cdot$ Benjamin Maasoumy ${ }^{1}$. \\ Torben Brod ${ }^{3}$ \\ ' Klinik für Gastroenterologie, Hepatologie und Endokrinologie, Medizinische Hochschule Hannover, \\ Hannover, Deutschland \\ ${ }^{2}$ Klinik für Kardiologie und Angiologie, Medizinische Hochschule Hannover, Hannover, Deutschland \\ ${ }^{3}$ Zentrale Notaufnahme und internistische IMC-Station, Medizinische Hochschule Hannover, Hannover, \\ Deutschland
}

Wir berichten über einen 43 Jahre alten Patienten, der sich im septischen Schock mit unspezifischer gastrointestinaler Symptomatik in der Notaufnahme vorstellte. Sonographie und CT erbrachten bei dem lebertransplantierten und mit mechanischer Mitralklappe versorgten Patienten keinen Infektfokus. Die Blutkulturen zeigten sich positiv für Listeria monocytogenes. Eine transösophageale Echokardiographie zeigte eine Prothesenendokarditis. Unter Ampicillin zeigte sich der Befund deutlich rückläufig.

Schlüsselwörter

Listeria monocytogenes · Prothesenendokarditis · Immunsuppression · Sepsis · Lebensmittelinfektion

\section{Anamnese}

Wir berichten über einen 43-jährigen männlichen Patienten, der sich mit Fieber, Schüttelfrost, Dysurie, abdominellen Schmerzen und produktivem Husten (klarer Auswurf) in der Notaufnahme vorstellte. Diese Beschwerden bestanden laut dem Patienten bereits seit einigen Tagen. Parallel berichtete der Patient, dass sich eine bekannte Herzinsuffizienz (NYHA III) in den letzten Tagen deutlich verschlechtert habe. Diarrhöen wurden von dem Patienten verneint. Auf explizites Nachfragen nach Konsum von rohen Lebensmitteln/ Fleisch gab der Patient an, regelmäßig Çiğ Köfte (rohe Hackfleischbällchen) zu sich zu nehmen. Eine differenzierte Anamnese war zu diesem Zeitpunkt aufgrund ausgeprägter Schmerzsymptomatik nicht möglich.
An relevanten Vordiagnosen war eine Lebertransplantation vor 30 Jahren bei Crigler-Najjar-Syndrom bekannt. Hierbei handelt es sich um einen seltenen Gendefekt mit verminderter Aktivität der Bilirubin-Glucuronosyl-Transferase, eine Hyperbilirubinämie ist die Folge. Unterschieden werden zwei Ausprägungen, Typ 1 mit komplettem Verlust der Enzymaktivität und Typ 2 mit partiellem Funktionsverlust. Folge kann ein Kernikterus sein. Eine Heilung des Typs 1 lässt sich durch Lebertransplantation erreichen. Im Verlauf ist eine von der Grunderkrankung unabhängige Rezirrhose des Transplantats aufgetreten sowie weiterhin ein mechanischer Mitralklappenersatz, der nach frustraner Mitralklappenrekonstruktion bei schwerer Mitralklappeninsuffizienz 4 Jahre vor Vorstellung implantiert worden war. 


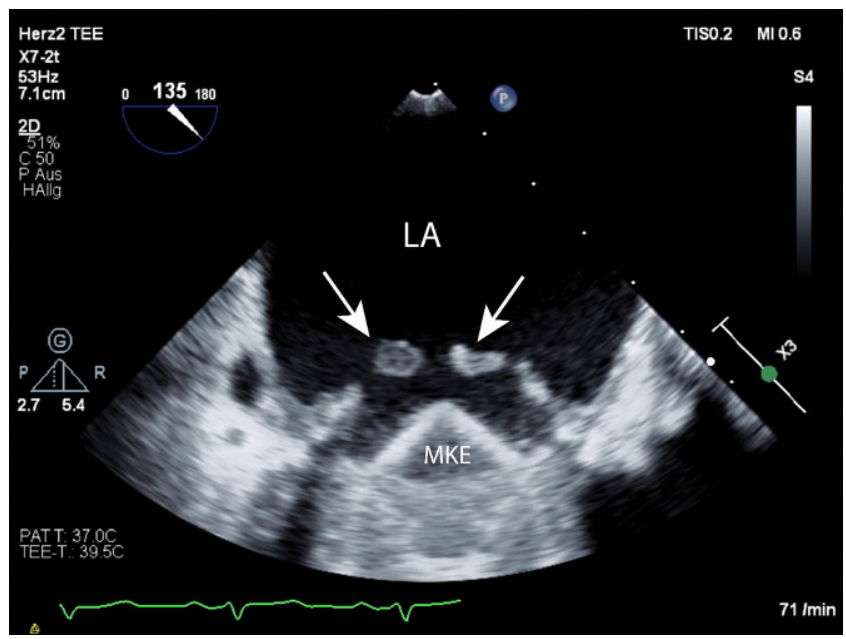

Abb. 14 Transösophageale Echokardiographie mit Darstellung von Vegetationen am Klappenring (Pfeile), LA linker Vorhof, MKE mechanischer Mitralklappenersatz

Zum Vorstellungszeitpunkt erfolgte die immunsuppressive Therapie mit Mycophenolatmofetil (MMF) $750 \mathrm{mg}$ zweimal täglich sowie Prednison $5 \mathrm{mg}$ einmal täglich. Die therapeutische Antikoagulation erfolgte mit Phenprocoumon.

\section{Befund}

Bei Vorstellung in der Notaufnahme zeigte sich ein positiver Schockindex ohne Sauerstoffpflichtigkeit des Patienten. Das C-reaktive Protein $(86,9 \mathrm{mg} / \mathrm{l})$ und das Prokalzitonin $(2,0 \mu \mathrm{g} / \mathrm{l})$ waren erhöht. Weiterhin imponierte ein erhöhtes Laktat $(2,9 \mathrm{mmol} / \mathrm{l})$ in der arteriellen BGA. Der durchgeführte Urinstatus zeigte sich unauffällig. Im klinischen Untersuchungsbefund zeigte sich eine Tachypnoe sowie auskultatorisch ein Befund passend zu einem mechanischen Klappenersatz, bei ansonsten unauffälligem Lungenauskultationsbefund. Die Oxygenierung war unter Raumluft suffizient. Der Volumenstatus zeigte sich eu- bis hypovolämisch. Der qSOFA-Score ergab 2 von 3 Punkten.

Nach Abnahme von 2 Blutkulturpaaren erfolgte die umgehende Einleitung einer Volumenersatztherapie sowie die empirische antiinfektive Therapie mittels $\mathrm{Pi}$ peracillin/Tazobactam 4 g/0,5 g. Weiterhin erfolgte bei persistierender Hypotonie eine niedrig dosierte Katecholamintherapie unter hämodynamischem Monitoring. Bei einem SOFA-Score von 9 Punkten wurde die Diagnose eines septischen Schocks gestellt. In Anbetracht der bestehenden Leberzirrhose waren auch die Diagnose- kriterien eines akut auf chronischen Leberversagens (ACLF) erfüllt.

Zur Fokussuche erfolgte zunächst die orientierende sonographische Untersuchung. Hierbei zeigten sich keine Pleuraergüsse oder Aszites. In der bekannt zirrhotisch umgebauten Transplantatleber konnte keine relevante Cholestase dargestellt werden. Bei septischem Schock mit im Vordergrund stehenden Bauchschmerzen erfolgte daher notfällig die computertomographische Bildgebung des Thorax und des Abdomens. Hier konnte kein eindeutiges Korrelat für die Beschwerdesymptomatik gefunden werden. In der transthorakalen Echokardiographie zeigte sich eine erhaltene LV- und RV-Funktion bei eingeschränktem Schallfenster.

\section{Diagnose}

\section{- Kunstklappenendokarditis mit septi- schen Schock durch Listeria monocy- togenes}

\section{Therapie und Verlauf}

Nach Aufnahme auf die internistische IMCStation zeigte sich der Patient im kurzfristigen Verlauf zunehmend schläfrig. Innerhalb der ersten $24 \mathrm{~h}$ bestand weiterhin ein steigender Katecholaminbedarf bei stagnierenden Infektparametern. Bei klinisch imponierender Somnolenz erfolgte die empirische Eskalation der antiinfektiven Medikation auf Meropenem und Linezolid (bei aus Voraufenthalten bekannter Besiedelung mit vancomycinresistenten Enterokokken). Das MMF wurde zu- nächst pausiert und das Prednison auf $15 \mathrm{mg}$ täglich gesteigert. Unter parallel forcierter Flüssigkeitsgabe gelang nach Eskalation der antiinfektiven Therapie eine langsame Reduktion der Katecholamingabe. Aus 3 der 4 abgenommenen Blutkulturen konnte Listeria monocytogenes nachgewiesen werden („time to positivity“ $22 \mathrm{~h}$ $49 \mathrm{~min}$ ). Es erfolgte die Umstellung auf eine Monotherapie mittels Ampicillin. Auf eine Kombination mit Gentamicin wurde aufgrund der chronischen Nierenfunktionseinschränkung zunächst verzichtet. In den folgenden Tagen zeigte sich der Katecholaminbedarf rasch rückläufig. Auch der klinische Zustand und die Laborchemie verbesserten sich kontinuierlich.

Aufgrund der eingeschränkten Beurteilbarkeit von transthorakal erfolgte eine transösophageale Echokardiographie. Hierbei zeigten sich 2 mobile Strukturen vorhofseitig $(7 \mathrm{~mm} \times 6 \mathrm{~mm}$ und $12 \mathrm{~mm} \times 3 \mathrm{~mm}$ ), passend zu einer Endokarditis des mechanischen Klappenersatzes (• Abb. 1).

Auf eine Liquorpunktion wurde bei rasch rückläufiger Somnolenz und ansonsten fehlender weiterer neurologischer Symptomatik aufgrund der therapeutischen Antikoagulation bei mechanischem Klappenersatz verzichtet.

In einer ersten transösophagealen Verlaufsechokardiographie ca. 1,5 Wochen nach Therapiebeginn zeigte sich eine deutliche Regredienz. Darstellbar war zu diesem Zeitpunkt noch eine Struktur $(8 \times 5 \mathrm{~mm})$.

\section{Diskussion}

Die Listeriose ist eine Lebensmittelinfektion, die in der Regel durch das grampositive Bakterium Listeria monocytogenes verursacht wird. Dieses wird unter anderem über rohes Fleisch und Rohmilchprodukte übertragen. Zuletzt ist es auch in Deutschland zu einem Ausbruch durch Lachs bzw. in Europa durch kontaminierte Blutwurst gekommen [3, 4]. Die Erkrankung ist für etwa $1 \%$ der lebensmittelassoziierten Infektionen verantwortlich [9]. Kritisch, besonders in der lebensmittelverarbeitenden Industrie, sind die Bildung von Biofilmen und Toleranzentwicklung gegenüber Händedesinfektionsmitteln und Salz. Auch besteht eine Vermehrungsfähigkeit bei $2-4^{\circ} \mathrm{C}$. Eine 
Übertragung ist nicht nur durch kontaminierte Lebensmittel, sondern auch direkt durch Mensch und Tier möglich. Typischerweise tritt die klinisch manifest werdende Listeriose in bestimmten Risikogruppen, wie z.B. immungeschwächten Personen, älteren Patienten, Neugeborenen oder Schwangeren, auf [5, 7]. Bei isolierter Gastroenteritis beträgt die Inkubationsdauer ca. 24h. Bei systemischen Infektionen kann jedoch eine deutlich größere Latenz auftreten, was die Diagnosestellung erheblich erschweren kann. Systemische Infektionen gehen dennoch häufig mit gastrointestinalen Symptomen einher, nahezu ein Viertel der Patienten leidet unter einer Diarrhö $[2,8]$. Die Dreimonatsmortalität systemischer Infektionen ist mit bis zu $45 \%$ hoch [2].. Grundsätzlich kann von einer Listerieninfektion jedoch jedes Organ betroffen sein. In der Vergangenheit sind bereits Endokarditiden an künstlichen Klappen beschrieben worden, wobei diese nur bei ca. $8 \%$ aller mit Listerien infizierten Personen auftreten und die Mortalität im Vergleich zu anderen Endokarditiserregern geringer zu sein scheint [6]. Die Therapie der Listeriose sollte mindestens 2 Wochen betragen, bei invasiven Infektionen, wie z. B. einer Endokarditis, sollte jedoch 4-6 Wochen therapiert werden, um Rezidiven vorzubeugen [10]. Die Therapie der ersten Wahl umfasst Ampicillin, wobei aufgrund der bakteriostatischen Wirkung eine Kombination mit einem Aminoglykosid, sofern hierfür keine Kontraindikationen vorliegen, zu diskutieren ist. Therapiemöglichkeiten zweiter Wahl sind u.a. Cotrimoxazol oder Fluorchinolone.

In dem hier vorgestellten Fall gelang der entscheidende Hinweis auf die Genese des septischen Zustands des Patienten über das Ergebnis der mikrobiologischen Diagnostik. Alle im Therapieverlauf gegebenen Antiinfektiva zeigen Wirksamkeit gegenüber Listeria monocytogenes. Aufgrund der um einige Tage verzögerten Vorstellung des Patienten nach Auftreten der Symptome lag zum Initiierungszeitpunkt der Therapie bereits eine invasive Erkrankung vor, sodass es trotz potenziell wirksamer antiinfektiver Therapie einige Tage bis zum signifikanten Ansprechen dauerte. Zur Differentialdiagnostik erfolgte der Ausschluss einer mechanischen Abflussstörung bzw. Cholangitis bei unspezifischer

Raw meat, lots of problems: rare infection in a patient after mechanical valve replacement and liver transplantation

We report about a 43-year-old man who presented to the emergency department in septic shock with nonspecific gastrointestinal symptoms. Sonography and computed tomography (CT) could not identify the location of the infection in the patient who had undergone liver transplantation and has a mechanical mitral valve. Blood cultures were positive for Listeria monocytogenes. Transesophageal echocardiography showed prosthetic endocarditis. The findings regressed markedly under ampicillin.

\section{Keywords}

Listeria monocytogenes · Prostetic valve endocarditis · Immunosuppression · Sepsis · Food-borne infections

Allgemeinsymptomatik. Wir möchten daher hervorheben, dass bei immunsupprimierten Patienten mit unspezifischer gastrointestinaler Symptomatik immer auch an eine Listerieninfektion gedacht werden sollte. Hierbei ist anzumerken, dass bei systemischer Listeriose Stuhlkulturen negativ ausfallen können und bei bestehendem Fieber immer Blutkulturen angelegt werden sollten. Immunsupprimierte Patienten sollten auf entsprechende Lebensmittel (auch bzgl. der Gefahr einer Hepatitis E) hingewiesen werden [1]. Im Falle unseres Patienten war aber auch die schnelle empirische antiinfektive Therapie essenziell aufgrund der vulnerablen Gesamtkonstitution mit Immunsuppression, Herzinsuffizienz und Leberzirrhose sowie dem Vorliegen eines ACLF auf dem Boden eines septischen Schocks. Hier darf die antiinfektive Therapie nicht verzögert werden.

Zuletzt möchten wir unterstreichen, dass der Verlauf einer Listerienendokarditis unter Mitbeteiligung eines mechanischen Klappenersatzes durchaus einer primär konservativen Therapie zugänglich ist (vgl. [6]) und nicht einer sofortigen operativen Revision bedarf.

\section{Fazit für die Praxis}

- Die Listeriose ist eine ernstzunehmende Lebensmittelinfektion mit potenziell hoher Mortalität.

- Risikofaktoren sind vor allem Schwangerschaft und Immunsuppression.

- Besonders bei diesen Patientengruppen sollte bei unspezifischer gastrointestinaler Symptomatik an eine (invasive) Listerieninfektion gedacht werden.

- Die antiinfektive Therapie der Wahl umfasst Ampicillin und ggf. ein Aminogly- kosid für mindestens 2 Wochen. Längere Therapiedauern sind je nach Fall nötig.

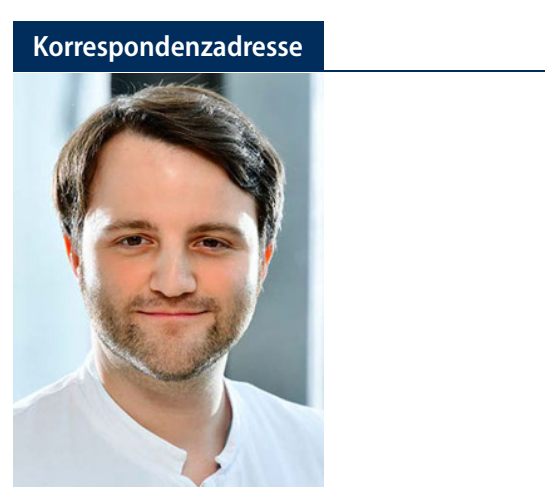

PD Dr. Benjamin Maasoumy

Klinik für Gastroenterologie, Hepatologie und Endokrinologie, Medizinische Hochschule Hannover

Carl-Neuberg-Str. 1, 30625 Hannover,

Deutschland

maasoumy.benjamin@mh-hannover.de

Funding. Open Access funding enabled and organized by Projekt DEAL.

\section{Einhaltung ethischer Richtlinien}

Interessenkonflikt. M. Dölle, S. Soltani, H. Wedemeyer, B. Maasoumy und T. Brod geben an, dass kein Interessenkonflikt besteht.

Für diesen Beitrag wurden von den Autoren keine Studien an Menschen oder Tieren durchgeführt. Für die aufgeführten Studien gelten die jeweils dort angegebenen ethischen Richtlinien. Für Bildmaterial oder anderweitige Angaben innerhalb des Manuskripts, über die Patienten zu identifizieren sind, liegt von ihnen und/oder ihren gesetzlichen Vertretern eine schriftliche Einwilligung vor.

Open Access. Dieser Artikel wird unter der Creative Commons Namensnennung 4.0 International Lizenz veröffentlicht, welche die Nutzung, Vervielfältigung, 
Bearbeitung, Verbreitung und Wiedergabe in jeglichem Medium und Format erlaubt, sofern Sie den/die ursprünglichen Autor(en) und die Quelle ordnungsgemäß nennen, einen Link zur Creative Commons Lizenz beifügen und angeben, ob Änderungen vorgenommen wurden.

Die in diesem Artikel enthaltenen Bilder und sonstiges Drittmaterial unterliegen ebenfalls der genannten Creative Commons Lizenz, sofern sich aus der Abbildungslegende nichts anderes ergibt. Sofern das betreffende Material nicht unter der genannten Creative Commons Lizenz steht und die betreffende Handlung nicht nach gesetzlichen Vorschriften erlaubt ist, ist für die oben aufgeführten Weiterverwendungen des Materials die Einwilligung des jeweiligen Rechteinhabers einzuholen.

Weitere Details zur Lizenz entnehmen Sie bitte der Lizenzinformation auf http://creativecommons.org/ licenses/by/4.0/deed.de.

\section{Literatur}

1. Behrendt P, Steinmann E, Manns MP, Wedemeyer H (2014) The impact of hepatitis e in the liver transplant setting. J Hepatol 61:1418-1429. https://doi.org/10.1016/j.jhep.2014.08.047

2. Charlier C, Perrodeau É, Leclercq A et al (2017) Clinical features and prognostic factors of listeriosis: the MONALISA national prospective cohort study. Lancet InfectDis 17:510-519. https:// doi.org/10.1016/S1473-3099(16)30521-7

3. Halbedel S, Wilking $\mathrm{H}$, Holzer A et al (2020) Large nationwide outbreak of invasive listeriosis associated with blood sausage, Germany, 2018-2019. Emerg Infect Dis 26:1456-1464. https://doi.org/ 10.3201/eid2607.200225

4. Robert Koch-Institut (2021) Mehrere ListerioseAusbrüche in Deutschland mit Hinweisen auf geräucherte oder gebeizte Lachsprodukte als Ursache von Infektionen. Epid Bull 7:3-9. https:// doi.org/10.25646/7851

5. Lamont RF, Sobel J, Mazaki-Tovi S et al (2011) Listeriosis in human pregnancy: a systematic review. J Perinat Med 39:227-236. https://doi.org/ 10.1515/JPM.2011.035

6. De Miguel-Yanes JM, González-Ramallo VJ, Pastor L (2004) Outcome of Listeria monocytogenes prosthetic valve endocarditis: as bad as it looks? Scand J Infect Dis 36:709-711. https://doi.org/10. 1080/00365540410021063a

7. de Noordhout CM, Devleesschauwer B, Angulo FJ et al (2014) The global burden of listeriosis: a systematic review and meta-analysis. Lancet Infect Dis 14:1073-1082. https://doi.org/10.1016/ S1473-3099(14)70870-9

8. Ooi ST, Lorber B (2005) Gastroenteritis due to Listeria monocytogenes. Clin Infect Dis 40:1327-1332. https://doi.org/10.1086/429324

9. Scallan E, Hoekstra RM, Angulo FJ et al (2011) Foodborne illness acquired in the United StatesMajor pathogens. Emerg Infect Dis 17:7-15. https://doi.org/10.3201/eid1701.P11101

10. RKI (Hrsg) Listeriose (RKI-Ratgeber). https://www. rki.de/DE/Content/Infekt/EpidBull/Merkblaetter/ Ratgeber_Listeriose.html.Zugegriffen:9.Mai 2021

\section{MED UPDATE SEMINARE} $2022 / 23$

\section{Endokrino Update 2022}

3. Endokrinologie-Update-Seminar

16.-17. September 2022

Berlin und Livestream

\section{Wiss. Leitung:}

Prof. Dr. Matthias Blüher, Leipzig

Prof. Dr. Jörg Bojunga, Frankfurt a. M.

Prof. Dr. Martin Fassnacht, Würzburg

Prof. Dr. Dr. Dagmar Führer, Essen

Unter der Schirmherrschaft der DGIM

www.endokrino-update.com

\section{Diabetes Update 2023}

18. Diabetologie-Update-Seminar

\section{4.-25. Februar 2023}

Mainz und Livestream

\section{Wiss. Leitung:}

Prof. Dr. Andreas Hamann, Bad Homburg

Dr. Helmut Kleinwechter, Kiel

Prof. Dr. Stephan Martin, Düsseldorf

Prof. Dr. Michael Stumvoll, Leipzig

Unter der Schirmherrschaft der DGIM

www.diabetes-update.com

Auskunft für alle Update-Seminare med update $\mathrm{GmbH}$

www.med-update.com

Tel.: 0611 - 736580

info@med-update.com

\section{Onko Update 2023}

18. Onkologie-Update-Seminar

20.-21. Januar 2023

Berlin und Livestream

27.-28. Februar 2023

Mainz und Livestream

Wiss. Leitung:

Prof. Dr. Lothar Kanz, Tübingen

Prof. Dr. Carsten Bokemeyer, Hamburg

Prof. Dr. Ralf Hofheinz, Mannheim

Prof. Dr. Andreas Neubauer, Marburg

Unter der Schirmherrschaft der DGIM

Mit Unterstützung der DKG

www.onko-update.com

\section{Gastro Update 2023}

30. Gastroenterologie-Update-Seminar

\section{0.-11. März 2023}

Mainz und Livestream

24.-25. März 2023

Berlin und Livestream

\section{Wiss. Leitung:}

Prof. Dr. Peter Layer, Hamburg

Prof. Dr. Thomas Berg, Leipzig

Prof. Dr. Andrea May, Wiesbaden

Prof. Dr. Andreas Stallmach, Jena

Unter der Schirmherrschaft der DGIM, ÖGGH

www.gastro-update.com

Auskunft für alle Update-Seminare: med update $\mathrm{GmbH}$ www.med-update.com Tel.: 0611 - 736580 info@med-update.com 